\title{
Instalação do sistema alternativo de irrigação por aspersão com garrafas PET em canteiro de terra na Fazenda Escola de São Luís (MA)
}

\author{
Installation of the alternative sprayer irrigation system with PET bottles on an \\ earth site at the Fazenda Escola de São Luís (MA)
}

\author{
Bárbara Noeme da Silva Souza ${ }^{1}$ (iD https://orcid.org/0000-0003-0395-3010 \\ Leuda Caroline Brito de Asevedo ${ }^{1}$ (i) https://orcid.org/0000-0001-5271-2200
}

\begin{abstract}
Resumo
A irrigação comercial no Brasil é reconhecidamente onerosa, obstáculo que restringe seu uso por grande parte dos agricultores familiares. Os sistemas alternativos de irrigação surgem como um marco da independência dos pequenos produtores em relação à equipagem e sistemas importados, dando-lhe uma opção viável de manter e aumentar sua produção, melhorando sua renda. O manejo da irrigação de uma cultura deve ser feito com base em critérios que possibilitem a aplicação de água no solo e promover produção ótima, do ponto de vista da economia. Este trabalho objetivou desenvolver um sistema de irrigação alternativo, direcionado para a produção familiar, demonstrar o funcionamento do sistema e determinar a vazão do sistema alternativo de irrigação. A área de estudo do sistema alternativo foi implantada no campus da Universidade Estadual do Maranhão - Campos São Luís, canteiro de 0,90mx15m área experimental da Fazenda Escola de São Luís. Trata-se de um sistema simples, feito a partir do aproveitamento de garrafas pet, cheias de água produzem uma irrigação no formato de chuva. Para manter o material suspenso, pode ser usado um piquete de madeira. Para confeccionar o aspersor foram feitos furos nas garrafas PET, utilizando-se uma agulha de costura quente, mangueiras, cola d'água. Para calcular a vazão dos aspersores, utilizou-se a formula simples da vazão. O sistema montado, apresentou uma boa eficiência, sem desperdiçar água ou causar erosões no canteiro, além de irrigar a área plantada. Com vazão encontrada de $0,0063 \mathrm{~L} \mathrm{~s}$. Por tanto, indicamos este sistema para pequenos produtores
\end{abstract}

Palavras-chave: Garrafas PET. Manejo de irrigação. Sustentabilidade.

\begin{abstract}
Commercial irrigation in Brazil is admittedly costly, an obstacle that restricts its use by most family farmers. Alternative irrigation systems emerge as a sign of the independence of small producers in rela-tion to equipment and imported systems, giving them a viable option to maintain and increase their pro-duction, improving their income. The management of a crop's irrigation must be based on criteria that allow the application of water to the soil and promote optimal production, from an economic point of view. This work aimed to develop an alternative irrigation system, aimed at family production, demons-trate the functioning of the system and determine the flow rate of the alternative irrigation system. The study area of the alternative system was set up on the campus of the State University of Maranhão - Campos São Luís, in a 0.90mx15 $m$ construction site in the experimental area of Fazenda Escola de São Luís. It is a simple system, made from the use of PET bottles, filled with water, producing irrigation in the form of rain. To keep the material suspended, a wooden picket can be used. To make the sprinkler, holes were made in the PET'S bottles, using a hot sewing needle, hoses, and wa-ter glue. To calculate the sprinkler flow rate, the simple flow formula was used. The assembled system showed good efficiency, without wasting water or causing erosion in the bed, in addition to irrigating the planted area. With a flow rate of $0.00063 \mathrm{~L} s$ found. Therefore, we recommend this system for small producers.
\end{abstract}

Keywords: PETS Bottles. Irrigation Management. Sustainability

1 Universidade Estadual do Maranhão, Centro de Ciências Agrarias, Economia Rural. Campus São Luís, Cidade Universitária Paulo VI, Av. Lourenço Vieira da Silva, 1000, Jardim São Cristóvão, 65.055-310. São Luís, MA, Brasil. Correspondência para/Correspondence to: B. N. S. SOUZA. E-mail: barbara_noeme03@hotmail.com. 


\section{Introdução}

A distribuição da vegetação sobre a superfície da terra é controlada mais pela disponibilidade de água do que qualquer outro fator. Cerca de 80 a $90 \%$ do peso fresco de uma planta herbácea e aproximadamente $50 \%$ das espécies lenhosas estão representados pela água. Além disso, ela é o solvente que permite que gases, minerais e outras substâncias possam penetrar nas células e fluir entre as mesmas e entre os vários órgãos do vegetal (Silva; Silva; Porto, 2014).

A disponibilidade de água é variável de acordo com as condições climáticas, tipo de solo e o seu manejo, sendo que esta é uma das principais responsáveis pelas flutuações na produção das culturas (Giasson; Merten, 2010). Além disso, o manejo dado ao solo é muito importante no aumento ou redução da disponibilidade hídrica dos solos.

O manejo da irrigação de uma cultura deve ser feito com base em critérios que possibilitem a aplicação de água no solo de forma a promover produção ótima, do ponto de vista econômico. A irrigação deve repor, ao solo, a quantidade de água retirada pela cultura e o momento de se irrigar é aquele no qual a disponibilidade de água no solo assume valor mínimo, abaixo do qual a planta começa a sentir os efeitos da restrição de água (Balbino, 2020). Portanto, o objetivo da irrigação é manter sempre um nível de água neste reservatório de forma a que as plantas possam crescer sem restrições. Para tanto, deve-se avaliar as condições de pressão, vazão e lâminas d'água aplicadas. Na realidade, a avaliação de sistemas de irrigação é um tema que os agricultores pouco têm dado importância. Mesmo tendo acesso à tecnologia, muitos não a utilizam de forma adequada, por falta de orientação e conhecimento.

Assim, os sistemas alternativos de irrigação surgem como um marco da independência dos pequenos produtores em relação à equipagem e sistemas importados, dando-lhe uma opção viável de manter e aumentar sua produção, melhorando sua renda, qualidade de vida e dando-lhe dignidade. Este trabalho objetivou desenvolver um sistema de irrigação alternativo, direcionado para a produção familiar, demonstrar o funcionamento do sistema e determinar a vazão do sistema alternativo de irrigação. O trabalho justifica-se pela importância que é atribuída a agricultura familiar que visa à geração de empregos e renda do homem no campo, a produção de alimentos e a busca por práticas agrícolas sustentáveis.

\section{Fundamentação Teórica}

Apesar do custo inicial de aquisição e instalação de sistemas de irrigação convencional ser elevado, principalmente para o pequeno agricultor, existem formas de adotar sistemas com menor custo, tornando-os mais acessíveis ao agricultor familiar. O uso de emissores artesanais é uma alternativa que diminui significativamente o custo de aquisição e manutenção de um sistema de irrigação.

De acordo com Coelho et al., 2012 várias recomendações de sistemas para pequenas 
áreas estão disponíveis, tais como o uso de irrigação por potes, irrigação tipo xique-xique, lowhead bubbler, sistema mandala, dentre outros. O uso de garrafas de plástico (PET) e outros objetos têm sido veiculados na mídia em sistemas de irrigação tipo micro aspersão com uso de cotonetes e dutos de água feitos de garrafas de plástico, como exemplo.

Coelho et al.,2014 afirma que quando tomados os devidos cuidados no uso da água, os sistemas de irrigação montados de material de baixo custo apresentam os mesmos efeitos dos sistemas convencionais sobre a produção de culturas de ciclo curto e perenes em áreas de agricultura familiar do semiárido.

De acordo com Nascimento (2017) as técnicas de irrigação de baixo custo visam atender a demanda hídrica das culturas, utilizando materiais não tradicionais em sistemas de irrigação, dando assim a possibilidade de o pequeno produtor conseguir fazer a aplicação de água investindo muito pouco em um sistema. Essas técnicas contemplam todos os métodos de irrigação e utilizam para isso materiais alternativos encontrados no cotidiano de um agricultor, trazendo alternativas eficientes atreladas ao uso racional de água.

A agricultura brasileira se destaca entre as maiores do mundo e representante uma fonte de alimentos e de matéria prima para muitos países. Nela estão presentes diversos modos de fazer agricultura, entre os quais a produção agrícola familiar, encontrada em extensas e importantes regiões do Brasil. A agricultura familiar no País é crescentemente uma forma social de produção reconhecida pela sociedade brasileira, por suas contribuições materiais e imateriais (Delgado et al., 2017)

Apesar dessa grande importância, historicamente no Brasil, os agricultores familiares sempre receberam pouco apoio do poder público para realizar suas atividades. Este segmento foi comparativamente negligenciado pelas diversas esferas governamentais ao longo do processo de modernização da agricultura brasileira, que se iniciou no final da Segunda Guerra Mundial (Castro, 2015).

A atividade agrícola familiar é ainda negativamente afetada pelo baixo nível de capacitação técnica e gerencial dos produtores. O frágil nível de sua organização dificulta uma maior inserção desse segmento produtivo no mercado e nas cadeias produtivas. A deficiência de coordenação e a desarticulação entre os distintos segmentos dos incipientes arranjos produtivos locais existentes impedem a plena ocupação dos espaços de valorização e competitividade dos produtos junto aos mercados regional e nacional (Silva; Guimarães Filho, 2006).

A agricultura irrigada se destaca entre os principais usos da água, sendo responsável por 47 da retirada dos mananciais e 69\% do consumo total no Brasil (Agência Nacional Das Águas, 2016). No Brasil, os cultivos irrigados representam cerca de $16 \%$ da demanda de alimentos e $35 \%$ do valor de produção (Silva et al., 2016). A técnica da irrigação é uma alternativa para suprir a carência hídrica das culturas em razão da desuniformidade da precipitação em algumas regiões (Carvalho et al, 2014). De acordo com Medeiros e Arruda (2016), a irrigação possui um papel de destaque, por ser um dos principais segmentos demandantes de água na sociedade e para o uso 
racional e sustentável deste recurso é necessário o conhecimento da demanda hídrica das principais culturas irrigadas regionalmente. A agricultura é a maior consumidora de água doce do planeta e a difusão de práticas sustentáveis de uso dos recursos hídricos é emergencial em regiões que já enfrentam episódios de estresse hídrico (United Nations Educational, Scientific and Cultural Organization, 2021).

\section{Materiais e Métodos}

A área de estudo do sistema alternativo foi implantada e monitorada no campus da Universidade Estadual do Maranhão - Campos São Luís (UEMA), em canteiro de 0,90mx15m na área experimental da Fazenda Escola de São Luís (FESL).

Os materiais utilizados na preparação do canteiro foram: enxada, gadanho, $45 \mathrm{~kg}$ de esterco bovino, pá, mudas de alface Americana, sementes de rúcula, semente de coentro verdão, garrafas PET fita dura poc, agulhas. O canteiro foi preparado 30 dias antes com a incorporação de $45 \mathrm{~kg}$ de esterco bovino e com a proximidade do plantio foi feito uma capina manual. Os canteiros foram demarcados com o auxílio da trena, no espaçamento de 0,30m entre linha e 0,25 entre plantas de alface; 0,25m entre linhas e 0,25m entre plantas de rúcula; 0,20m entre linhas de coentro. Na cultura do coentro o plantio é continuo, não sendo adotado o espaçamento dentre plantas. Foram montados os aspersores e para confeccionar foram feitos furos nas garrafas PET, utilizando-se uma agulha de costura quente, mangueiras, cola d'água. No meio da garrafa foi realizado a abertura de um orifício, levando em consideração o diâmetro da mangueira utilizada. Trata-se de um sistema simples, feito a partir do aproveitamento de garrafas PET, que perfuradas e cheias de água produzem uma irrigação no formato de chuva. Para manter o material suspenso, pode ser usado um piquete de madeira, para calcular a vazão dos aspersores, utilizou-se a formula simples da vazão. Foram realizadas visitas diárias ao canteiro para monitorar a eficiência do sistema utilizado.

\section{Resultados e Discussão}

A disponibilidade de uma adequada série de dados de vazão é essencial em grande parte dos estudos hidrológicos. A noção real do volume de água afluente a determinada seção se mostra imprescindível em qualquer etapa de planejamento e gestão de recursos hídricos, desde a compatibilização entre oferta e demanda (World Meteorological Organization, 2010; Tomkins, 2014; Coxon et al., 2015).

A irrigação por aspersão é a aplicação da água ao solo resulta da subdivisão de um jato d'água lançado sob pressão no ar atmosférico, através de simples orifícios. Para calcular a vazão dos aspersores, utilizou-se a formula simples da vazão:

$$
\mathrm{Q}=\mathrm{V}
$$

V- Velocidade T- tempo 
Com os valores das vazões dos aspersores coletados em campo, verificou-se que o modelo desenvolvido apresenta vazão suficiente para suprir a demanda hídrica da maioria das culturas que são possíveis de serem irrigadas por aspersores, conforme apresenta o Quadro 1. Foram realizadas quatro medidas em 120 segundos.

Quadro 1 - Demanda da vazão no sistema de irrigação.

\begin{tabular}{c|c}
\hline Medida 1 & $0,14 \mathrm{~L}$ \\
\hline Medida 2 & $0,23 \mathrm{~L}$ \\
\hline Medida 3 & $0,025 \mathrm{~L}$ \\
\hline Medida 4 & $0,16 \mathrm{~L}$ \\
\hline A vazão encontrada & $0,0063 \mathrm{~L} / \mathrm{s}$ \\
\hline
\end{tabular}

Fonte: Elaborado pelo autor (2021),com base em demonstrar o funcionamento e determinar a vazão, a demanda hídrica do sistema alternativo de irrigação.

Inicialmente foram apresentadas algumas dificuldades na confecção do sistema de irrigação utilizado. O primeiro sistema montado apresentou muitas falhas no seu funcionamento, devido ao tamanho e posição dos furos realizados nas garrafas, que acabaram transformando um sistema alternativo em um tremendo prejuízo, por causar do grande desperdício de água e por criar várias erosões no canteiro. De acordo com Camargo (2016) quantidade de água que a planta recebe reflete no seu desenvolvimento, pois a irrigação está associada à capacidade de retenção de água do solo e necessidade hídrica da planta. Em contrapartida, com o aperfeiçoamento da técnica, o sistema montado, apresentou uma boa eficiência, funcionando do jeito que esperávamos, sem desperdiçar água ou causar erosões no canteiro, além de irrigar a área plantada (Figura 1), mostrando-se apto para ser utilizado pelos pequenos produtores.

De acordo com o autor Dillon et al.,1995 afirma que pequenos projetos podem gerar baixos custos e permitem uma melhor gestão dos recursos hídricos, principalmente por apresentar um baixo custo de aquisição, sendo necessário apenas de mangueiras, agulhas de costura, cola d'agua e das pet's que são facilmente encontradas em pontos de coleta ou em lugares de alimentação, como restaurantes, lanchonetes, entre outros.

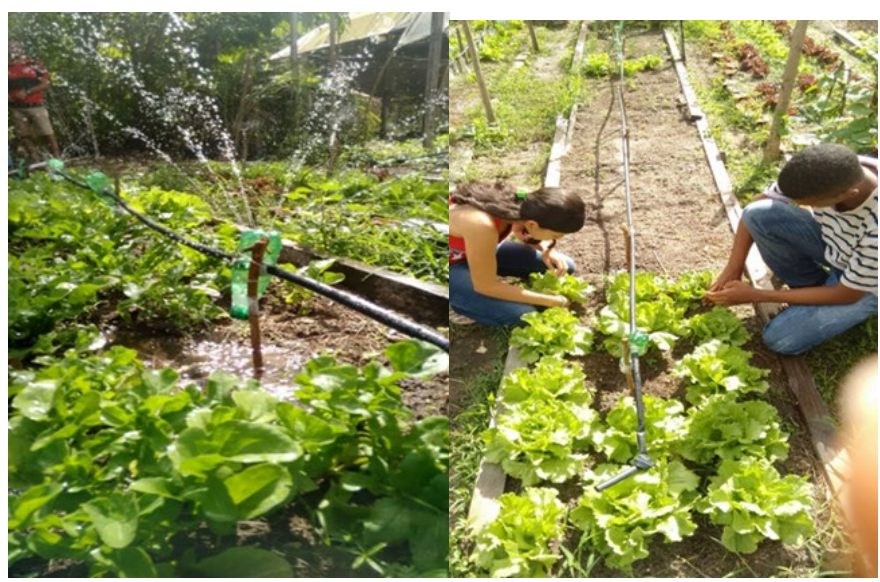

Figura 1 - Sistema de irrigação alternativa com garrafa PET.

Fonte: Acervo pessoal dos autores (2021). 


\section{Considerações Finais}

Portanto, indicamos este sistema para pequenos produtores e alertamos para a importância de realizar os furos nas garrafas de forma correta, visando o mínimo desperdício de água e a máxima eficiência de irrigação. O manejo correto em um sistema de irrigação é importante para que o agricultor tenha sucesso na produção. Em virtude disso, a escolha de um sistema de irrigação simplificado, eficiente e de fácil acesso ao pequeno agricultor é um fator determinante para que o mesmo tenha maior margem de lucro.

\section{Referências}

Agência Nacional Das Águas. Conjuntura dos recursos hídricos no Brasil: Informe 2016. Brasília, DF: ANA, 2016. Disponível em: http://www.snirh.gov.br/portal/snirh/centrais-deconteudos/conjuntura-dos-recursos-hidricos. Acesso em: 11 abr. 2019

Balbino, N. S. Conflito de uso entre abastecimento público e irrigação associado à crise hídrica na Bacia do Alto Descoberto, Distrito Federal. Brasília: UNB, 2020. Disponível em: https://repositorio.unb.br/handle/10482/40818. Acesso em: 12 set. 2021

Camargo, D. C. Manejo da Irrigação: como, quando e quanto irrigar? Fortaleza: INOVAGRI/IFCE, 2016.

Carvalho, I. R. et al . Desempenho agronômico de híbridos de milho em ambiente irrigado e sequeiro. Enciclopédia Biosfera, v. 10, n. 18, p. 1144-1153, 2014.

Castro, C. N. Desafios da agricultura familiar: o caso da assistência técnica e extensão rural. Boletim Regional, Urbano e Ambiental, v. 12, p. 51-52, 2015.

Coelho, E. F.et al. Sistemas de irrigação para agricultura familiar. Cruz das Almas: Embrapa Informação Tecnológica, 2012.

Coelho, E. F.et al. Sistemas e manejo de irrigação de baixo custo para agricultura familiar. Cruz das Almas: Embrapa Mandioca e Fruticultura, 2014.

Delgado, G. C. et al. Agricultura familiar brasileira: desafios e perspectivas de futuro. Brasília: Ministério do Desenvolvimento Agrário, 2017. v. 470.

Dillon, J. C. et al. Nutricional value of the alga Spirulina. In: Simopoulos, A. P. (ed.). World review of nutrition and dietético: plants in humannutrition. Basel: Karger, 1995. v. 77, p. 32-46.

Giasson, E.; Merten, G. H. Caracterização dos fatores de produção da unidade de produção agrícola: gestão e planejamento de unidades de produção agrícola. Rio Grande do Sul: Universidade Aberta do Brasil, 2010. p. 37-52.

Medeiros, G. A.; Arruda, F. B. Avaliação do consumo de água pela irrigação: Parametrização do coeficiente de cultura em função do desenvolvimento da planta. Sínteses: Revista Eletrônica do SIMTEC, n. 1, p. 5-13, 2016.

Nascimento, F. F. L. Sistemas e manejo de irrigação de baixo custo. Cruz das Almas: Bahia, 2017.Disponivel:

https://repositorio.unilab.edu.br/jspui/bitstream/123456789/1886/1/CLICE\%20DE\%20ARA\%C3\% 9AJO\%20MENDON\%C3\%87A\%20TCC.pdf. Acesso em: 22 set. 2020.

Silva, P. C. G.; Guimarães Filho, C. Eixo tecnológico da e corregião nordeste. In: Sousa, I. S. F. (ed.). Agricultura familiar na dinâmica da pesquisa agropecuária. Brasília, DF: Embrapa Informação Tecnológica, 2006. Cap. 3, p.109-123.

Silva, T. O.; Silva, M. P. P.; Porto, K. C. Briófitas de afloramentos rochosos do Estado de Pernambuco. Salvador: Universidade Federal da Bahia, 2014. 
Silva, et al. Agricultura irrigada no contexto amazônico: uma abordagem sistemática do uso da água em uma horticultura no município de Altamira-PA. Revista Internacional de Ciências, v. 6, n. 1, p. 29-43, 2016.

Tomkins, K. M. Uncertainty in streamflow rating curves: methods, controls and consequences. Hydrological processes, v. 28, n. 3, p. 464-481, 2014.

United Nations Educational, Scientific and Cultural Organization. The United Nations world water development report 2020: water and climate change. Paris: Unesco, c2021.

World Meteorological Organization. Manual on Stream Gauging. Geneva: Chairperson Publications Board, 2010.

Como citar este artigo/How to cite this article

SOUZA, B N S.; ASEVEDO, L. C. B. Instalação do sistema alternativo de irrigação por aspersão com garrafas pets em canteiro de terra na fazenda escola de São Luís (MA). Sustentabilidade: Diálogos Interdisciplinares, v. 2, e215522, 2021. https://doi.org/10.24220/2675$\underline{7885 \mathrm{v} 2 \mathrm{e} 2021 \mathrm{a} 5522}$

Recebido em 7 de dezembro de 2021 e aprovado em 14 de dezembro de 2021. 